\title{
TRAMAS DA PSICOLOGIA DA SAÚDE NO BRASIL: UMA ANÁLISE COM FERRAMENTAS DA TEORIA ATOR-REDE
}

THE WEFT OF HEALTH PSYCHOLOGY IN BRAZIL: AN ANALYSIS WITH TOOLS OF THE ACTOR-NETWORK THEORY

\section{TRAMAS DE LA PSICOLOGÍA DE LA SALUD EN BRASIL: UN ANÁLISIS CON HERRAMIENTAS DE LA TEORÍA ACTOR-RED}

\author{
Maria Eugênia Costa Machado* \\ Luciana Kind*
}

\begin{abstract}
RESUMO
Este artigo apresenta um estudo sobre a constituição da Psicologia da Saúde no Brasil, por meio de ferramentas teórico-metodológicas da teoria ator-rede. Como recurso metodológico, foram investigados os programas de pós-graduação no Brasil em Psicologia da Saúde e a Associação Brasileira de Psicologia da Saúde. Buscou-se ainda apresentar e discutir o modo como a Psicologia da Saúde aparece nas produções desenvolvidas nos programas de pós-graduação. $\mathrm{O}$ estudo mostra que a Psicologia da Saúde no Brasil tem se configurado como subcampo da Psicologia. Nos diferentes lugares investigados por esta pesquisa, foram encontradas formas distintas de configuração da Psicologia da Saúde, as quais estão permeadas de controvérsias, debates e disputas. O estudo evidencia pontos de convergências que sinalizam para uma singularidade, em especial a questão da interdisciplinaridade, a busca do status de cientificidade, a construção de redes de associações e o enfoque na prevenção e promoção da saúde.
\end{abstract}

Palavras-chave: Psicologia da Saúde. Teoria ator-rede. Interdisciplinaridade.

\begin{abstract}
This article presents a study about the development of Health Psychology in Brazil through the theoretical and methodological tools from the ActorNetwork theory. Both, the postgraduate programs in Health Psychology in Brazil and the Brazilian Association for Health Psychology have been investigated as methodological resources. It was also meant to present and discuss how Health Psychology appears in the works developed in the postgraduate programs. The present study shows that Health Psychology in Brazil has been conceived as a subfield of Psychology. In the different

Texto recebido em 15 de maio de 2019 e aprovado para publicação em 12 de agosto de 2019.

*Doutora em Psicologia, coordenadora do Curso de Psicologia no Centro Universitário de Belo Horizonte (Uni BH).

*Doutora em Saúde Coletiva (IMS/UERJ), coordenadora do Programa de Pós-Graduação em Psicologia na Pontifícia Universidade Católica de Minas Gerais (PUC Minas). Endereço: Avenida Itaú, 525, 1º subsolo - Dom Cabral, Belo Horizonte-MG, Brasil. CEP: 30535-012. E-mail: lukind@gmail.com.
\end{abstract}


places where investigations took place along this research, different forms of configuration of Health Psychology were found, which are permeated by controversies, debates, and disputes. The study shows points of convergence that signal towards a singularity, especially the matter of interdisciplinarity, the search for the scientificity status, the construction of networks of associations and the focus on prevention and the promotion of health.

Keywords: Health Psychology. Actor-network theory. Interdisciplinarity.

\section{RESUMEN}

Este artículo presenta un estudio sobre la constitución de la Psicología de la Salud en Brasil por medio de herramientas teórico-metodológicas de la teoría actor-red. Como recurso metodológico, fueron investigados los programas de postgrado en Brasil en Psicología de la Salud y la Asociación Brasileña de Psicología de la Salud. También se buscó presentar y discutir la forma en que la psicología de la salud aparece en las producciones desarrolladas en los programas de postgrado. El estudio muestra que la psicología de la salud en Brasil se ha establecido como un subcampo de la psicología. En los diferentes lugares investigados por este estudio fueron encontradas distintas formas de configuración de la psicología de la salud, que están impregnadas de controversias, debates y disparates. El estudio destaca puntos de convergencia que señalan a una singularidad, en particular la cuestión de la interdisciplinariedad, la búsqueda del estatus de la ciencia, la construcción de redes de asociaciones y el enfoque en la prevención y promoción de la salud.

Palabras-clave: Psicología dela Salud. Teoría actor-red. Interdisciplinariedad.

\section{INTRODUÇÃO}

terminologia "Psicologia da Saúde" (Health Psychology) foi denominada
pela Associação Americana de Psicologia (APA) no fim da década de 1970
início da de 1980. Relaciona-se com a criação de uma força-tarefa sobre de pesquisar a função do comportamento nas doenças físicas e na manutenção da saúde (APA Task Force on Health Research, 1976). Mas foi em 1978 que a associação instituiu a Divisão 38, cuja conceituação revela que as contribuições da Psicologia da Saúde perpassam por "avaliações do papel do comportamento na etiologia e no tratamento da doença, elaboração de prognósticos dos comportamentos prejudiciais para a saúde, promoção de comportamentos saudáveis e prevenção do aparecimento das doenças" (Almondes, 2013, p. 648). Essa construção foi resultado de debates antecipados por Engel (1977) 
e Matarazzo (1980), em artigos científicos. Engel (1977) apresentou oposição à psiquiatria biológica vigente, convocando a um modelo biopsicossocial de atuação nessa área, expressão que foi consolidada nas Ciências da Saúde de variadas maneiras.

Ribeiro (2011) afirma que a primeira definição de Psicologia da Saúde foi proposta por Stone, em 1979, quando ele alegou que essa é

Qualquer aplicação científica ou profissional de conceitos e métodos psicológicos, a todas as situações próprias do campo da saúde, não apenas nos cuidados de saúde, mas também na saúde pública, educação para a saúde, planificação da saúde, financiamento, legislação, etc. (Ribeiro, 2011, p. 24).

No entanto foi a contribuição de Matarazzo (1980) que sustentou a definição da APA. Para o autor, a Psicologia da Saúde serve à análise dos modos de se pensar a saúde com foco nos serviços e políticas, constituindo-se, portanto, como um sistema de saber/fazer. Trata-se de um "conjunto de contribuições educacionais, científicas e profissionais específicas da Psicologia, utilizadas para a promoção e manutenção da saúde, prevenção e tratamento das doenças, identificação da etiologia e diagnóstico" (Matarazzo, 1980, p. 815).

Nessa direção, a definição da APA afirma, de acordo com Almondes (2013, p. 648), que a "Psicologia da saúde constitui estratégia de atuação que agrega o conhecimento educacional, científico e profissional da Psicologia para as discussões do processo de saúde-doença”.

A partir da consideração de que a definição de Matarazzo é muito ampla, uma vez que abrange tudo o que é possível fazer no sistema de saúde em geral, durante a Conferência de Arden House, realizada em Nova Iorque, cujo objetivo era propor recomendações acerca da formação na Psicologia da Saúde, afirmouse que esta seria uma área genérica da Psicologia, que teria o próprio corpo teórico e científico, que se diferenciaria de outras áreas da Psicologia.

Esses embates indicam que ainda não se conseguiu estabelecer uma definição muito clara que permeia os debates acerca da Psicologia da Saúde. Sebastiani e Maia (2005) defendem que a Psicologia da Saúde nasceu para dar resposta às demandas nos níveis primário, secundário e terciário de atenção à saúde, pautando no modelo biopsicossocial e ambiental, numa perspectiva interdisciplinar.

Para Jacó-Vilela e Degani-Carneiro (2015), há um duplo sentido na expressão Psicologia da Saúde: tanto é uma vertente dentre as diferentes interfaces da Psicologia com a Saúde quanto uma denominação que abrange o conjunto de relaçōes que a Psicologia mantém nas instituiçōes de saúde. Almeida e Malagris 
(2011, p. 191) reforçam, nessa direção, que as funções da Psicologia da Saúde "estão se expandido à medida que o campo amadurece". As Psicologias da Saúde, portanto, são diferentes, são enacted distintamente onde quer que esteja sendo praticada (hospital, empresa, organização não governamental [ONG], clínica, escola, etc.). Se olharmos de perto para cada uma das Psicologias da Saúde, veremos que chegam a ser contraditórias, não se reduzindo a um único objeto e que são múltiplas.

Com a disseminação dos contornos da Psicologia da Saúde, houve distintas reverberaçôes quanto ao reconhecimento desta no contexto brasileiro, podendose dizer que ocorreram variaçóes de como a terminologia chegou a nosso país. No Brasil, a interlocução com as diretrizes da APA observadas por alguns autores (Castro \& Bornholdt, 2004; Gorayeb, 2010) fez-se em meio a variadas denominaçôes, a partir de experiências científicas e profissionais distintas, que produziram derivações como Psicologia Social da Saúde, Psicologia Hospitalar, Psiconcologia, Psicossomática e Psicologia Médica, somente para citar algumas, sendo que a especialização mais difundida é a Psicologia Hospitalar. Nesse ponto, vale destacar a fundação, em 1997, da Sociedade Brasileira de Psicologia Hospitalar (SBPH), responsável pela execução de congressos, produções científicas e conquista no Conselho Federal de Psicologia (CFP) do título de especialista em Psicologia Hospitalar.

Castro e Bornholdt (2004) afirmam que a tão difundida especialização em Psicologia Hospitalar no Brasil é inexistente em outros países, ainda que se reconheça a equivalência com a denominação Psicologia da Saúde em nosso país. Nas palavras das autoras, "A aproximação ao que seria no Brasil a Psicologia Hospitalar é denominada Psicologia da Saúde em outros países" (Castro \& Bornholdt, 2004, p. 48). No entanto, saúde e hospital são conceitos distintos; o conceito de saúde se relaciona às funções orgânicas, psíquicas e sociais, e as intervenções ocorrem nas atenções primária, secundária e terciária (hospitalar); enquanto o hospital equivale a uma instituição na qual as pessoas doentes são tratadas, estejam elas internadas ou não. Portanto as intervençôes ocorrem a nível secundário e terciário, já que, quando nos referimos ao hospital, automaticamente pensamos em algum tipo de doença já instalada. Para as autoras, então, a Psicologia Hospitalar deveria estar inclusa na Psicologia da Saúde, sendo "mais adequado referir-se à Psicologia no contexto hospitalar como um trabalho que faz parte da Psicologia da Saúde" (Castro \& Bornholdt, 2004, p. 55).

Ao tomar a interface entre Psicologia e Saúde e a multiplicidade de fatores que advém dessa relação no cenário brasileiro, questiona-se acerca das condições de produção da Psicologia da Saúde no Brasil. Nesse intuito, este trabalho tem como objetivo descrever a constituição da Psicologia da Saúde no Brasil por 
meio de ferramentas teórico-metodológicas da Teoria Ator-Rede (TAR). Para tal, a Psicologia da Saúde não será tomada como um objeto singular e definido, pronto e acabado, que pode ser interpretado, analisado e olhado de várias perspectivas, mas sim alguma coisa que pode ser construída, performada de maneiras distintas. Importa, aqui, levar em consideração os actantes, pois, para que os objetos sejam produzidos, há pessoas e coisas, cabendo ao pesquisador atentar ou deixar em segundo plano inúmeros elementos heterogêneos que constituem o fazer, o construir dos objetos.

Actante é o ator da expressão ator-rede. Latour (2000) chega a preferir utilizar a expressão actante, e não ator, para se referir aos integrantes da rede, porque a palavra ator se encontra associada aos humanos. Lemos (2013, p. 42) afirma que o actante "é o mediador, o articulador que fará a conexão e montará a rede nele mesmo e fora dele em associação com outros. Ele é que 'faz fazer". Ou seja, é o que/quem atua ou move a ação. Assim, o questionamento que interessa nessa investigação científica tem a ver com o modo como os objetos são manuseados, ou seja, como é que tais objetos se relacionam com as práticas.

\section{METODOLOGIA}

Pesquisar, com base nos pressupostos da TAR, incide numa tomada de posição diante de caixas-pretas, "seja para acompanhar as controvérsias que as encerram, seja para acompanhar as controvérsias que as reabrem” (Melo, 2007). É tarefa do pesquisador, da pesquisadora permitir aos interessados o acompanhamento de controvérsias acerca de como os atores se interligam uns aos outros, como se articulam em redes, por meios de ligações (Latour, 2000).

A proposta da TAR é seguir os envolvidos no desenvolvimento de artefatos, acompanhando as discussões. A ideia é que entremos nas atividades internas da ciência para depois sairmos e explicarmos como tudo funciona, ou seja, descrever como a produção dos elementos está sendo construída. Como Latour (2000, p. 17) nos convida a pensar, a entrada "no mundo da ciência [. . . ] será pela porta de trás, da ciência em construção, e não pela entrada mais grandiosa da ciência acabada". A TAR busca identificar as associaçôes entre os atores, que são vistos como mediadores ou intermediários, merecendo destaque as redes que se formam com a circulação da ação entre eles, que poderá ou não se tornar uma caixa-preta. Examinar uma caixa-preta significa atentar para as controvérsias que ocorrem entre os múltiplos atores.

Nessa direção, cabe ao pesquisador, baseado no que elege como material empírico, decidir a forma de considerá-los para, consequentemente, apresentálos, sendo que a postura do pesquisador ator-rede deve ser simétrica. Mol (2007) 
enfatiza que o pesquisador é quem decide o que fica dentro e o que fica de fora de sua pesquisa. Essas decisões é que constituem o percurso metodológico delineado para o desenvolvimento do estudo proposto pelo pesquisador.

Para a descrição dos programas de pós-graduação no Brasil em Psicologia da Saúde, realizamos duas buscas: referentes às avaliações de 2010 e 2013, cuja intenção foi acompanhar essa rede após os resultados das avaliações trienais da Coordenação de Aperfeiçoamento de Pessoal de Nível Superior (Capes), 2007/2009 e 2010/2012, o que compreende os resultados mais próximos ao tempo do desenvolvimento de nossa pesquisa. Tais buscas foram realizadas no site da Capes. No desenvolvimento da pesquisa, fizemos o levantamento de todos os programas e suas respectivas notas. Em seguida, entramos no site dos programas identificados com o intuito de selecionar as informações específicas, como área de concentração e linhas de pesquisa, que pudessem subsidiar nossa análise. Quando encontramos os programas cuja titulação era Psicologia da Saúde ou que houvesse alguma conexão, via áreas de concentração ou linhas de pesquisa, com tal terminologia, selecionamos as informações detalhadas dos programas e, posteriormente, produzimos a tessitura da rede de construção dessa Psicologia. Também buscamos nos Lattes dos docentes as informaçôes relativas a suas trajetórias acadêmicas, as quais compuseram nossa descrição sobre os atores humanos associados aos programas.

No decorrer do estudo, foram feitas ainda constantes investigações na página da Associação Brasileira de Psicologia da Saúde para acompanhar os movimentos da entidade. Com base nas informações recolhidas no site sobre quem eram os atores humanos envolvidos com a Associação, buscamos os dados nos respectivos currículos Lattes.

Partindo do pressuposto de Latour (2000, p. 90), de que os textos, embora possam parecer "aborrecidos e sem vida, de um ponto de vista superficial", carregam um potencial caso o leitor se proponha a desvendar os desafios colocados, é que assumimos a ideia de tecer uma rede. Buscamos as (re)associações dos textos que tratam da Psicologia da Saúde no Brasil nos periódicos associados aos programas de Pós-graduação descritos anteriormente. Partimos da ideia de que as produções encontradas também são actantes envolvidos no desenvolvimento dessa Psicologia.

No campo metodológico, buscamos apresentar e discutir o modo como a Psicologia da Saúde aparece nas produçôes desenvolvidas nos programas de pós-graduação. Nossa escolha por esse caminho está ancorada na ideia da complexidade que seria mapearmos todos as produções nacionais sobre a Psicologia da Saúde. Como critério básico, selecionamos estudos que discutem o 
desenvolvimento da Psicologia da Saúde no Brasil. Inicialmente realizamos uma busca no banco de teses e dissertações da Coordenação de Aperfeiçoamento de Pessoal de Nível Superior (CAPES) utilizando o descritor "Psicologia da Saúde". Em seguida, utilizamos os seguintes filtros:
a) instituição: Universidade Católica Dom Bosco;
b) nome do programa: Psicologia;
c) área concentração: Psicologia da Saúde.

Nessa busca, encontramos 50 dissertações de mestrado e 1 tese de doutorado. O mesmo critério foi utilizado para a segunda instituição:
a) instituição: Universidade Metodista de São Paulo;
b) nome do programa: Psicologia da Saúde;
c) área concentração: Psicologia da Saúde.

Nessa busca, encontramos 48 dissertações de mestrado.

A pesquisa foi igualmente realizada para a Universidade Federal de Santa Maria (UFSM), na qual foram encontradas 79 dissertaçôes de mestrado:
a) instituição: Universidade Federal de Santa Maria;
b) nome do programa: Psicologia;
c) área concentração: Psicologia da Saúde.

Após a leitura dos títulos dos 178 trabalhos encontrados, identificamos uma dissertação de mestrado, defendida na UFSM, com estudo sobre o desenvolvimento da Psicologia da Saúde no Brasil. Os demais trabalhos são, em sua maioria, estudos empíricos que versam sobre perspectivas clínicas, qualidade de vida, promoção da saúde e questões de saúde e doença.

Dado o número reduzido de trabalhos encontrados, partimos para uma busca de artigos publicados nos periódicos vinculados aos programas. Igualmente, utilizamos o descritor "Psicologia da Saúde" nos campos de busca das referidas revistas. Na revista Mudanças - Psicologia da Saúde, foram localizados nove artigos. Desses, após leitura dos títulos e resumos, apenas um foi selecionado, conforme critério. Na Revista Psicologia e Saúde, foram localizados 66 artigos, sendo selecionado apenas um, após leitura dos títulos e resumos.

Nesse ponto, vale destacar que, quando nos propomos a pesquisar um assunto, ou seja, quando abrimos as caixas-pretas, estamos (re)abrindo controvérsias e 
colocando à prova a dureza dos fatos, pois estes são fabricados. A TAR é, portanto, uma oportunidade de examinar os fatos duros, promovendo um movimento para dissipar preconceitos, a partir do acompanhamento das controvérsias, para originar novas inscrições que possam ser confrontadas com as, até então, construídas, para eventualmente criar objetos.

\section{RESULTADOS E DISCUSSÕES}

Ao buscarmos o (des)envolvimento das Psicologias da Saúde no Brasil, foi possível encontrar pontos de congruência que nos convidam a esboçar uma singularidade que envolve essa Psicologia, em especial o enfoque na prevenção, proteção, promoção e manutenção da saúde, para além do foco nas doenças. Como bem ressaltam Teixeira e Leal (1990, p. 454), "em Psicologia da Saúde entrecruzam-se inexoravelmente o discurso médico e o discurso psicológico". Por isso a interdisciplinaridade na Psicologia da Saúde assume um papel de destaque.

\subsection{Pós-graduação e Psicologia da Saúde no Brasil}

Os programas de pós-graduação no Brasil relativos explicitamente à Psicologia da Saúde, seja como programa, área de concentração ou linha de pesquisa, são oito atualmente, mas o programa da Universidade de Brasília (UnB) intitulado "Processos de desenvolvimento humano e saúde" parece não oferecer mais vagas para a linha de pesquisa denominada "Psicologia da Saúde"; em contrapartida, o programa Psicologia Clínica e Cultura, vinculado à mesma instituição, passou a oferecer a linha de pesquisa intitulada "Psicologia da Saúde e processos clínicos". Portanto consideramos que atualmente há sete ${ }^{3}$ programas que ofertam, explicitamente, vagas para trabalhos sobre a Psicologia da Saúde.

No total de 92 programas existentes em 2016, 4 oferecem mestrado e doutorado, 2 oferecem apenas o mestrado e 1 oferece o mestrado profissional. $\mathrm{O}$ mestrado profissional é uma nova tendência que "busca colocar o conhecimento como tributário da aplicação [. . . ] embora não exista claramente um norte bem definido sobre como se dará a convivência destes tipos de formatação da pósgraduação" (Cirani, Campanario, \& Silva, 2015, p. 166).

O programa mais antigo é o da Universidade Metodista de São Paulo (UMESP), que foi criado em 1978, e o mais recente é o da FPS, que iniciou em 2016. No contexto atual, temos a terminologia Psicologia da Saúde expressa em três programas, cinco áreas de concentração e parcialmente em duas linhas de pesquisa. Nenhum programa, dos que foram avaliados em 2016, aumentou 3 Universidade Metodista de São Paulo (UMESP), Universidade Católica Dom Bosco (UCDB), Pontifícia Universidade Católica de Goiás (PUC Goiás), Universidade de Brasília (UnB), Universidade Federal de Santa Maria (UFSM), Universidade Federal da Paraíba (UFPB) e Faculdade Pernambucana de Saúde (FPS). 
a nota, quando comparados com as avaliações anteriores realizada pela Capes. Os programas da Universidade Católica Dom Bosco (UCDB) e da PUC Goiás abriram na década de 1990, respectivamente em 1997 e em 1999.

Quando buscamos nas páginas dos programas de pós-graduação os docentes que participam destes, encontramos o de total 94 atores. Em 2015, o número total de professores era de 58, o que corrobora os achados de Cirani, Campanario e Silva (2015), de que o corpo docente da pós-graduação stricto sensu tem crescido sistematicamente no Brasil.

Como apontamos, o programa de pós-graduação mais antigo entre os que olharemos de perto é o da UMESP. Inicialmente, a formação oferecida foi a do mestrado; a formação em doutoramento é recente, iniciada em 2014. Até o ano de 1996, era o único programa de Psicologia da Saúde no Brasil. Apesar de o Programa ter iniciado suas atividades em 1978, foi em 1983 que passou a denominar-se Psicologia da Saúde, termo que, na época, causou estranhamento, pois "era pouco usual, na Psicologia brasileira, esta nomenclatura para nomear estudos do processo saúde e doença fora do âmbito da Saúde Mental" (Jesus \& Rezende, 2006, p. 122). A definição assumida pelo programa na sua apresentação se refere à de Matarazzo (1980), que é, ainda hoje, a mais conhecida. A aposta do programa é de que o interesse pela Psicologia da Saúde envolve profissionais da área da saúde, não se restringindo aos psicólogos. Portanto a concepção de Psicologia da Saúde assumida pelo Programa consegue abarcar outros profissionais de áreas afins à Psicologia, o que acentua uma perspectiva interdisciplinar e multiprofissional. Ribeiro (2011), no livro Psicologia da Saúde: teoria, intervenção e pesquisa, afirma que a formação interdisciplinar em Psicologia da Saúde é essencial e que "o vocabulário médico e o conhecimento do sistema de saúde são essenciais para trabalhar em vários contextos de investigação e de prática" (Ribeiro, 2011, p. 42). No mesmo livro, Alves, Ernesto, Silva, Lima, \& Eulálio (2011) ressaltam que a interlocução multiprofissional é fundamental entre os profissionais da saúde.

Poucos professores são associados da ABPSA, enquanto vários docentes estão envolvidos diretamente com a revista Mudanças - Psicologia da Saúde, seja como revisor ou revisora e, ou, membro do corpo editorial, seja como autor ou coautor de artigos. A revista é uma publicação sob a responsabilidade do Programa de Pós-Graduação em Psicologia da Saúde da UMESP. Atualmente, a avaliação do periódico em Psicologia é baixa, sem fator de impacto. A equipe editorial em sua totalidade é composta por atores da UMESP.

O Programa de Pós-Graduação da UCDB em Psicologia, nota 4 desde o início de nosso mapeamento, conta com uma área de concentração e duas linhas 
de pesquisa. É possível, nesse programa, desenvolver pesquisas que envolvam um dos níveis de atenção de saúde (primária, secundária e terciária - hospitalar), além da possibilidade de se estudarem os sistemas de saúde, levando em consideração as perspectivas multidisciplinar, interdisciplinar e transdisciplinar.

Praticamente todos os docentes estão vinculados ao periódico do programa, a Revista Psicologia e Saúde, como revisor ou revisora, e, ou, como editor, editora, e, ou, coautor ou coautora. Esse periódico é uma publicação do Programa de Mestrado e Doutorado em Psicologia da UCDB. Com essa proposta, as possibilidades de se publicar nessa revista são vastas, pois há aceitação de trabalhos que envolvam todos os três níveis de atenção à saúde (primária, secundária e terciária, ou hospitalar), cuja articulação pode-se entrelaçar à cultura, à política, à epidemiologia, à qualidade de vida e aos processos sociais que envolvam tanto a saúde quanto as políticas sociais.

A Revista Psicologia e Saúde melhorou seu Qualis possui uma boa classificação, alçando estratos mais altos na avaliação do Qualis Periódicos. O comitê editorial é composto em sua maioria por atores humanos da UCDB, enquanto o conselho editorial é todo composto por pesquisadores que não têm vínculos explícitos com a instituição; há vários de instituições internacionais. Esse cenário nos mostra que o periódico está almejando alcançar a excelência nacional.

O Programa de Pós-Graduação em Psicologia da UFSM, assim como o da UCDB, apresenta a Psicologia da Saúde como área de concentração. A nota do programa é 3. Como apontamos anteriormente, o programa iniciou suas atividades em 2009. Entre os sete programas que se articulam com a Psicologia da Saúde, via nome, área de concentração ou linha de pesquisa, existentes no Brasil, o da UFSM foi o quarto a ser criado; e entre os três que estamos utilizando em nosso estudo, é o mais recente.

Sobre os atores humanos das instituições que compuseram nosso estudo, podemos dizer que estes se identificam com inúmeras linhas de pesquisa e áreas de atuação. Além disso, suas formaçōes e trajetórias acadêmicas são as mais diversas possíveis, fato que também caracteriza a interdisciplinaridade da Psicologia da Saúde, pois, em maior ou menor grau (a UMESP e a UCDB mais que a UFSM), recebe atores de áreas afins à Psicologia, seja nos congressos ou nos programas de pós-graduação, nos quais encontramos descrições bastante amplas e interdisciplinares. Consideramos ser também relevante apontar que, para sobressair-se no cenário nacional da Psicologia da Saúde, é preciso fazer parcerias internacionais, tal como a UMESP e a UCDB têm feito, pois percebemos que os actantes que se encontram associados a outros, quer seja nacional, quer seja internacional, tem se destacado no cenário nacional da Psicologia da Saúde. 
Diante do exposto, percebemos que a UMESP é a instituição que mais se destaca no cenário brasileiro, mas ainda de uma forma tímida, pois a Associação Brasileira de Psicologia da Saúde (ABPSA) como um ator não humano, por exemplo, alcança um número reduzido de atores humanos; já a Revista Mudanças tem conseguido se manter em excelência nacional, e o Programa de Pós-Graduação, que é interdisciplinar, tem crescido com a proposta de acolher estudos cuja demarcação esteja na perspectiva biopsicossocial. A UCDB se apresenta como um ator que tem se esforçado para se destacar a partir da Revista Psicologia e Saúde e do Programa de Pós-Graduação. O periódico acolhe um leque de possibilidades de divulgação de estudos nas áreas da Psicologia e da Saúde, enquanto o Programa de Pós-Graduação, também interdisciplinar, tem um foco bastante voltado para as políticas públicas. A UFSM é a instituição que menos se destaca no país, tendo em vista que o Programa de Pós-Graduação, que não indica ser interdisciplinar, é o mais recente, o qual ainda oferece apenas o mestrado como titulação e não tem periódico; aceita o desenvolvimento de instrumentos de avaliação psicológica, estudos em bioética e estudos sobre psicanálise; o foco do programa é psicossocial e também voltado para as políticas públicas; as associações estabelecidas até o momento se relacionam com a ABPSA e com a Revista Mudanças.

\subsection{Ciência em ação}

Nas informações que recolhemos na página da ABPSA, encontramos o esforço de narrativa histórica de fundação de um novo modo de produzir conhecimento em torno da Psicologia da Saúde atrelado aos objetivos da Associação.

A fundação da Associação decorreu de uma resolução do Colegiado do Programa de Pós-Graduação em Psicologia da Saúde da UMESP, que funciona no interior do Estado de São Paulo. Para a reunião de fundação, estiveram representadas seis instituições de ensino superior brasileiro e uma portuguesa, incluindo cinco programas de pós-graduação.

Três programas de pós-graduação estavam representados: o Programa de Pós-Graduação em Psicologia da Saúde da UMESP; o Programa de PósGraduação em Psicologia da Universidade Federal de Uberlândia; e o Programa de Pós-graduação em Psicologia Escolar e do Desenvolvimento Humano da Universidade de São Paulo. ${ }^{4}$ Dessas instituições, duas (a UMESP e a UFU), naquele momento, tinham a formação stricto sensu específica em Psicologia da Saúde. Atualmente, somente a primeira continua com a formação stricto sensu específica em Psicologia da Saúde.

4 Todas as informaçôes acerca dos atores foram retiradas dos currículos Lattes destes. Todas os dados lançados no Lattes são de responsabilidade dos pesquisadores. 
A UMESP contava com a área de concentração Psicologia da Saúde, assim como ainda é nos dias atuais, além de ser o nome do Programa. A UFU tinha a Psicologia da Saúde como uma linha de pesquisa. Atualmente essa instituição conta com a linha Processos Psicossociais em Saúde e Educação. Conseguimos identificar essa mudança na UFU, porque fizemos um mapeamento dos programas de pós-graduação brasileiros no ano de 2013 e outro no ano de 2015 para acompanharmos a avaliação destes pela CAPES no período correspondente ao de nossa pesquisa.

Os dois programas de pós-graduação que estavam representados pelos docentes ausentes foram o Programa de Pós-Graduação em Gerontologia da Universidade Estadual de Campinas (Unicamp) e o Programa de Pós-Graduação em Psicologia da Universidade de Fortaleza (Unifor). Sobre as instituições representadas pelos ausentes, encontramos duas brasileiras e uma estrangeira, Faculdade de Engenharia Química de Lorena (Faenquil), Escola de Engenharia de Lorena/ Universidade de São Paulo (EEL/USP) e Instituto Superior Miguel Torga (ISMT - Portugal). Em nenhum desses programas há a área de concentração, tampouco a linha de pesquisa "Psicologia da Saúde".

A primeira diretoria executiva eleita, também na ocasião da fundação, foi composta por nove nomes, todos docentes de programas de pós-graduação, representantes de seis instituiçõos brasileiras de ensino superior. ${ }^{5}$ Mais uma vez, vemos uma predominância do projeto científico. Sabemos que docentes de programas de pós-graduação se envolvem em projetos de pesquisa, em editoração de revistas científicas, participam de eventos científicos, entre outros afazeres que são característicos do âmbito científico.

$\mathrm{Na}$ composição atual, a diretoria conta com 16 participantes. Há uma representação institucional estrangeira (Universidade de Algarve) e oito instituições de ensino superior brasileiras representadas, cuja predominância é da UMESP. No total são dez pessoas ligadas à essa universidade direta ou indiretamente. Essa composição aponta que as associaçôes, do ponto de vista do social tal como é proposto por Latour (2000), concentram-se e estão ligadas predominantemente na UMESP, pois, como dito anteriormente, a ABPSA foi criada com o protagonismo evidente do Programa de Pós-Graduação em Psicologia da Saúde dessa universidade, além de ser a responsável pela Revista Mudanças, que é um periódico direcionado para a Psicologia da Saúde.

Dos 16 participantes da atual diretoria, cinco estavam na primeira gestão; apenas uma não está diretamente ligada à UMESP. Além da UMESP, encontramos

\footnotetext{
5 Universidade Metodista de São Paulo (UMESP), Universidade Federal de Uberlândia (UFU), Universidade de Fortaleza (Unifor), Universidade Estadual de Campinas (Unicamp), Faculdade de Engenharia Química de Lorena (Faenquil) e Escola de Engenharia de Lorena/Universidade de São Paulo (EEL/SP).
} 
docentes de outras sete instituições de ensino superior ${ }^{6}$ e um representante de prefeitura municipal. Do total de participantes, doze são docentes de programas de pós-graduação stricto sensu, enquanto os demais ou lecionam na graduação, ou estão envolvidos com pesquisas voluntariamente, ou estão cursando a pósgraduação stricto sensu.

A ABPSA, como toda entidade científica, promove periodicamente congressos em sua área, a Psicologia da Saúde.

\subsection{Produções da Psicologia da Saúde no Brasil}

Alves et al. (2011), em artigo intitulado Psicologia da saúde: abrangência e diversidade teórica, buscou conhecer os discursos da Psicologia da Saúde em revistas com publicação nacional. A justificativa para a proposição do estudo perpassa pela ideia de que a Psicologia da Saúde é um tema novo, amplo, não consensual e que não existe um acordo em relação à delimitação e abrangência.

Especificamente sobre a abrangência, as autoras chamam a atenção para o fato de que há um "diálogo travado entre pesquisadores [. . .] sobre a existência de uma diferença formal entre a psicologia clínica e a psicologia da saúde" (Alves et al., 2011, p. 2), sendo que os questionamentos dizem respeito à hierarquia de pertencimento: a Psicologia da Saúde é mais ampla do que a Psicologia Clínica e a última é uma de suas intervenções ou é o contrário, a Psicologia Clínica é que é mais abrangente do que a Psicologia da Saúde?

Segundo as autoras, estudiosos estadunidenses, europeus e cubanos, como Matarazzo, Godoy, Simon, Teixeira e Lovelle, são defensores de que a Psicologia da Saúde é uma disciplina de abrangência ampla, considerando sua autonomia em relação a clínica (Alves et al., 2011). As autoras chamam atenção para o fato de que, na perspectiva dos autores mencionados, a Psicologia da Saúde se centra em intervenções voltadas para os diversos níveis de atenção (primária, secundária e terciária), com práticas atreladas à saúde geral.

Nos discursos nacionais, as autoras recorrem a pesquisadoras renomadas, como Spink (1992; 2011), que produz constante diálogo entre a Psicologia Social e a Saúde. Alves et al. (2011) marcam o entendimento dessa autora acerca da amplitude da Psicologia Social como uma área capaz de abarcar aplicações de saúde.

Conforme a Psicologia passa a se interessar pela promoção da saúde e pela prevenção de doenças, inicia-se um deslocamento do modelo clínico tradicional 6 Universidade de Taubaté (Unitau), Universidade Paulista (Unip), Pontifícia Universidade Católica do Rio Grande do Sul (PUCRS), Universidade do Vale do Paraíba (Univap), Universidade Federal de Santa Maria (UFSM), Universidade Federal do Rio Grande do Sul (UFRGS), Universidade de São Paulo (USP), Universidade Braz Cubas (UBC), Centro Universitário das Faculdades Metropolitanas Unidas (FMU) e Centro Universitário São Camilo-SP. 
de diagnosticar e tratar. Com o surgimento de programas como o Programa de Saúde da Família (PSF), Núcleo de Apoio à Saúde da Família (NASF), Centro de Referência da Assistência Social (CRAS) e Centro de Referência Especializado da Assistência Social (CREAS), entre outros, foi necessária a constituição de equipes multidisciplinares, de modo que o trabalho do psicólogo nessas equipes exige o estabelecimento de um diálogo interdisciplinar (Alves et al., 2011).

As discussões apresentadas pelas autoras nos permitem identificar certa "disputa" quanto à denominação e caracterização do que, de fato, é Psicologia da Saúde. A imprecisão toma acento quando as autoras apresentam estudos que buscam delimitar a Psicologia da Saúde com base na Psicologia Social ou na Psicologia Clínica. Além disso, percebemos que há uma polissemia quanto à terminologia do que venha a ser denominado Psicologia da Saúde. Em relação a esse fato, temos observado que se trata de uma característica marcante dessa Psicologia.

Quanto à regionalização, apesar de a Psicologia da Saúde estar, como apontado por Alves et al. (2011), consolidada em certas regióes, em alguns países da América Latina, a formação ainda se concentra mais nos cenários da pósgraduação; além disso, os estudos são precários para possibilitar intervençóes incisivas que considerem as especificidades e contextos socioeconômicos de cada região.

O estudo de Alves et al. (2011) aponta a existência de uma Psicologia da Saúde que, embora se constitua de modo multidisciplinar, ainda tem seus conceitos com pouca capilaridade nos meios acadêmicos, marcando uma dificuldade em produzir consensos bem como uma maior entrada nos espaços de prática profissional. As autoras marcam, nas consideraçôes finais, que se trata de uma Psicologia "abrangente e difusa", reforçando a necessidade de constante discussão buscando o mapeamento e consolidação da Psicologia da Saúde.

O artigo de Daneluci (2013), intitulado Psicologia e saúde como campo de interrogações, busca, por meio de uma revisão de literatura, problematizar a relação entre a Psicologia e a Saúde. Para tal, a autora apresenta os seguintes questionamentos: há diferenças entre as áreas da Psicologia e da Saúde? A existência de uma remete a outra? Por que ainda falamos da inserção do psicólogo na área da saúde? A defesa da autora é de que houve "mudanças no interior da Psicologia, tanto teóricas como de modelos e locais de atuação, com foco na área da Saúde", culminando em "fragmentações no interior do próprio campo da Psicologia, o que gerou diversas expressóes e até especializações, como a Psicologia da Saúde e Hospitalar" (Daneluci, 2013, p. 18). Nessa direção, verificamos um posicionamento explícito "na contramão dessas divisões, questionando o 
que compóe a Psicologia em sua singularidade, sem adjetivos e preposições" (Daneluci, 2013, p. 18).

A autora sustenta que a "disputa" de terminologias, como Psicologia da Saúde, Psicologia na Saúde, Psicologia Hospitalar, etc., seria desnecessária, pois na concepção da Psicologia, já há a pressuposição do conceito de Saúde, assim como no conceito de Saúde já existe implicada a concepção da Psicologia, haja vista que o conceito de saúde integra as perspectivas psicológica, biológica e social.

Após delimitar as áreas da Psicologia e Saúde, construindo seus distanciamentos e aproximações, Daneluci (2013) se questiona acerca da interdependência entre os campos, ou seja, se a existência de um campo remete à existência do outro. Para tanto, a autora apresenta o início da Psicologia da Saúde na década de 1970 com a definição de Matarazzo (1980), como um marco histórico. A definição desse autor, conforme argumenta o artigo, passou a valorizar os fenômenos sociais no processo saúde-doença e a enfatizar a atenção primária. A autora recorre a Spink para afirmar que "a Psicologia da Saúde surge como novo campo de saber, diferenciando-se da Psicologia Clínica tradicional” (Daneluci, 2013, p. 20).

No que diz respeito à classificação da Psicologia da Saúde, verificamos um ponto de divergência entre os estudos. Enquanto Alves et al. (2011), pautadas, especialmente, pelo diálogo teórico, apresentam a Psicologia da Saúde como uma área; Daneluci (2013) utiliza argumentos teóricos para desconstruir a necessidade de uma classificação. A imprecisão sobre o (não) lugar em que a Psicologia da Saúde ocupa parece fortalecer a lógica contrária à sua consolidação. Ao mesmo tempo, parece-nos que tomar a Psicologia da Saúde como um subcampo está mais próximo do entendimento pautado na atuação profissional. Não obstante, Daneluci (2013) busca trabalhar, respectivamente, a atuação da Psicologia no campo da Saúde e a Psicologia da ou na Saúde como uma possibilidade de intervenção. Vale ressaltar que Daneluci (2013) defende a redundância em falar Psicologia da Saúde.

No que tange aos argumentos utilizados pelas autoras, parece-nos que o texto de Alves et al. (2011) caminha em direção à validação da Psicologia da Saúde. Para isso, sistematiza o conceito de Psicologia da Saúde, utilizando um recorte mais amplo até chegar no nível nacional, passando pela América Latina. Há uma tentativa de construir, historicamente, o modo como a Psicologia da Saúde foi se consolidando, seja como um saber ou como um instrumento de intervenção. Já Daneluci (2013) usa os argumentos teóricos para produzir uma crítica à Psicologia da Saúde. O texto recorre a autores da Psicologia e da Saúde para marcar as interrogações e contradições que estão presentes no campo da Psicologia 
da Saúde. Com um olhar pautado para o cenário da prática de atuação, a autora parece desconsiderar a Psicologia da Saúde como um saber. Embora Daneluci (2013) utilize autores que também estão presentes nos outros textos, essa autora parece servir-se da discussão em Psicologia da Saúde com foco no profissional da Psicologia. O olhar da autora para a Psicologia da Saúde parece caminhar em direção à construção da singularidade do psicólogo, da psicóloga, defendendo, portanto, que a fragmentação produzida pela classificação da Psicologia da Saúde contribuiria para uma difícil produção de singularidade.

Ademais, vale destacar que os estudos carregam elementos para pensarmos a rede de (re)associações da Psicologia da Saúde no Brasil emergindo como actantes no desenvolvimento dessa Psicologia. A definição de Matarazzo sobre a Psicologia da Saúde ainda é um ponto de partida para o desenvolvimento das discussões dessa Psicologia no Brasil. Apesar disso, há controvérsias estabelecidas, como sinaliza o texto de Daneluci (2013), indicando ser possível desconsiderar a definição da Psicologia da Saúde.

\section{CONSIDERAÇÕES FINAIS}

Consideramos ser importante ressaltar que, apesar de a prática da Psicologia na área da Saúde ser antiga, por ter começado nas instituições de saúde antes mesmo da regulamentação da profissão em nosso país, a Psicologia da Saúde, enquanto nomenclatura, é recente no cenário brasileiro. Nesse sentido, embora tenha uma associação científica, programas de pós-graduação e publicações mantêm a diversidade de abordagens e formas de atuação, reproduzindo a diversidade de origem que continua presente na Psicologia. A Psicologia da Saúde ainda tem um longo caminho a percorrer para sua constituição como uma área, bem demarcada dentro da Psicologia, pois ainda não há unanimidade quanto à clareza do que corresponda exatamente a essa Psicologia.

O estudo mostra que a Psicologia da Saúde no Brasil tem se configurado como subcampo. Nos diferentes lugares que seguimos nesta pesquisa, encontramos formas distintas de configuração da Psicologia da Saúde, as quais estão permeadas de controvérsias, debates e disputas. Ora percebemos o projeto científico da Psicologia da Saúde distante do projeto profissional, ora os percebemos próximos. Há trabalhos (dissertações, teses e artigos) que discutem a prática da Psicologia da Saúde sem sequer tocar em tal Psicologia como ciência, mas também há estudos, poucos, que se interessam pela Psicologia da Saúde como uma ciência. Ora os temas dos congressos dizem respeito à Psicologia da Saúde como "ciência", ora como prática profissional.

Se desmembramos a terminologia "Psicologia da Saúde" em "Psicologia" e 
"Saúde", teremos multiplicidade em ambas, "Psicologias" e "Saúdes", pois são muitas as psicologias e muitas as saúdes (física, social, psicológica, econômica, política, etc.). Portanto falar em Psicologia da Saúde é falar em Psicologias da Saúde, é falar em multiplicidade, é falar em heterogeneidade. É falar de diferença. No primeiro artigo, de Alves et al. (2011), que utilizamos na descrição da rede de textos, assistimos à disputa de terminologias, abordagens, teorias e instrumentos nos quais as Psicologias da Saúde se encontram no País. Em contrapartida, no segundo texto, de Daneluci (2013), a defesa da autora é de que é desnecessário falar em Psicologia da Saúde ou na Saúde, já que a relação entre Psicologia e Saúde é complementar. Já na dissertação de Silva (2010), que foi o terceiro texto utilizado por nós, a autora considera que a Psicologia da Saúde tem uma base teórica suficiente para compreender a saúde dos indivíduos e das populações. Enquanto actantes, esses textos nos mostram que as diferenças entre as Psicologias da Saúde perpassam por disputas que ultrapassam a nomenclatura do que de fato pode ser considerado como Psicologia da Saúde.

Ademais, na perspectiva da TAR, verifica-se que o conceito da Psicologia da Saúde ainda não pode ser considerado uma caixa-preta; ainda não é um intermediário, pois não transporta um significado sem modificação; e ainda não há somente reprodução, ocorrendo produção de significado. $O$ que percebemos foram mediadores, ou seja, atores que traduzem a multiplicidade das terminologias da Psicologia da Saúde, transformando e traduzindo o significado de tal Psicologia. 


\section{REFERÊNCIAS}

Almeida, R. A. \& Malagris, L. E. N. (2011). A prática da psicologia da saúde. Revista da SBPH, 14(2), 183-202. Recuperado a partir de http://pepsic. bvsalud.org/scielo.php?script=sci_arttext\&pid=S1516-08582011000200012

Almondes, K. M. (2013). Psicologia da saúde e cronobiologia: diálogo possível? Psicologia: Ciência e Profissão, 33(3), 646-655. Recuperado a partir de http:// dx.doi.org/10.1590/S1414-98932013000300010

Alves, R. F., Ernesto, M. V., Silva, R. P da, Lima, A. G. B., \& Eulálio, M. do C. (2011). Psicologia da saúde: abrangência e diversidade teórica. Mudanças: PsicologiadaSaúde, 19(1-2), 1-10. Recuperadoa partirdehttps://www.metodista. br/revistas/revistasims/index.php/MUD/article/viewFile/2479/2914

Alves, R. F. \& Eulálio, M. do C. (2011). Abrangência e níveis de aplicação da Psicologia da saúde. In R. F. Alves (Org.), Psicologia da Saúde: teoria, intervenção e pesquisa (pp. 65-88). Campina Grande: Eduepb.

Castro, E. K. \& Bornholdt, E. (2004). Psicologia da Saúde x Psicologia Hospitalar: definições e possibilidades de inserção profissional. Psicologia Ciência e Profissão, 24(3), 48-57. Recuperado a partir de http://dx.doi.org/10.1590/S141498932004000300007

Cirani, C. B. S., Campanario, M. de A., \& Silva, H. H. M. (2015). A evolução do ensino da pós-graduação senso estrito no Brasil: análise exploratória e proposições para pesquisa. Avaliação, 20(1), 163-187.

Contributions of Psychology to health research: patterns, problems and potentials (1976). American Psychologist, 31(4), 263-274.

Daneluci, R. de C. (2013). Psicologia e saúde como campo de interrogaçóes. Revista Psicologia e Saúde, 5(1), 18-24. Recuperado a partir de http://pepsic. bvsalud.org/scielo.php?script=sci_arttext\&pid=S2177-093X2013000100004 \&lng=pt\&tlng=pt

Engel, G. (1977). The need for a new medical model: a challenge for biomedicine. Science, 196(4286), 129-136.

Gorayeb, R. (2010). Psicologia da Saúde no Brasil. Psicologia: Teoria e Pesquisa, 26 (Número Especial), 115-122. Recuperado a partir de http://dx.doi. org/10.1590/S0102-37722010000500010 
Jacó-Vilela, A, M. \& Degani-Carneiro, F. (2015). Psicologia e Saúde no Brasil: interfaces históricas. Revista Tempos Gerais, 4(2), 144-161. Recuperado a partir de http://www.seer.ufsj.edu.br/index.php/temposgerais/article/ view/1438/1079

Jesus, S. N. \& Rezende, M. M. (2006). Atualidades em Psicologia da saúde: colaborações Brasil e Portugal. Mudanças: Psicologia da Saúde, 14(2), 121-125. Recuperado a partir de https://www.metodista.br/revistas/revistas-ims/index. php/MUD/article/viewFile/642/642

Keef, F. \& Blumenthal, J. (2004). Health Psychology: what will the future bring? Health Psychology, 23(2), 156-157. Recuperado a partir de http://dx.doi. org/10.1037/0278-6133.23.2.156

Kerbauy, R. R. (2002). Comportamento e saúde: doenças e desafios. Psicologia USP, 13(1), 11-28. Recuperado a partir de http://dx.doi.org/10.1590/S010365642002000100002

Kind, L. (2010). Psicologia e saúde: a produção de estilos de pensamento. Latin-American Journal of Fundamental Psychopathology Online, 7(1), 113129. Recuperado a partir de http://www.psicopatologiafundamental.org.br/ uploads/files/latin_american/v7_n1/psicologia_e_saude.pdf

Latour, B. (2000). Ciência em ação: como seguir cientistas e engenheiros sociedade afora. I. C. Benedetti (Trad.). São Paulo: Editora Unesp.

Lemos, A. (2013). A comunicação das coisas: teoria ator-rede e cibercultura. São Paulo: Annablume.

Matarazzo, J. (1980). Behavioral health and behavioral medicine: frontiers for a new health psychology. American Psychologist, 35(9), 807-817.

Melo, M. F. A. Q. (2007). Seguindo as pipas com a metodologia da TAR. Revista do Departamento de Psicologia, 19(1), 169-185. Recuperado a partir de http:// dx.doi.org/10.1590/S0104-80232007000100013

Mol, A. (2007). Política ontológica: algumas ideias e várias perguntas. In J. A. Nunes \& R. Roque (Orgs.), Objectos impuros: experiências em estudos sociais da ciência. (pp. 22-40). Porto: Afrontamento.

Ribeiro, J. L. P. (2011). A Psicologia da saúde. In R. F., Alves (Org.), Psicologia da Saúde: teoria, intervenção e pesquisa. Campina Grande: Eduepb. 
Sebastiani, R. W. \& Maia, E. M. C. (2005). Contribuições da Psicologia da Saúde-Hospitalar na atenção ao paciente cirúrgico. Acta Cirurgica Brasileira, 20(Supl. 1), 50-55. Recuperado a partir de http://dx.doi.org/10.1590/S010286502005000700010

Silva, R. R. da. (2010). O perfil de saúde de estudantes universitários: um estudo sob o enfoque da Psicologia da Saúde. (Dissertação de Mestrado). Universidade Federal de Santa Maria, Programa de Pós-graduação em Psicologia, Santa Maria. Recuperado a partir de http://200.18.45.28/sites/ppgp/images/ documentos/texto\%209.pdf

Spink, M. J. P. (1992). Psicologia da saúde: a estruturação de um novo campo de saber. In F. C. B. Campos (Org.), Psicologia e saúde: repensando práticas. (pp. 11-23). São Paulo: Hucitec.

Spink, M. J. P. (2011). Psicologia social e saúde: práticas, saberes e sentidos. Petrópolis: Vozes.

Stone, G. C. (1979). Psychology and health system. In G. C. Stone, F. Cohen, N. E. Adler (Orgs.), Health Psychology. (pp. 47-75). San Francisco: Jossey-Bass.

Teixeira, J. A. C. \& Leal, I. (1990). Psicologia da saúde: contexto e intervenção. Análise Psicológica, 8(4), 453-458. 